\title{
Dynamical excitation of maxon and roton modes in a Rydberg-Dressed Bose-Einstein Condensate
}

\author{
Gary McCormack ${ }^{1}$, Rejish $\mathrm{Nath}^{2}$ and Weibin $\mathrm{Li}^{1}$ \\ ${ }^{1}$ School of Physics and Astronomy, and Centre for the Mathematics and Theoretical \\ Physics of Quantum Non-Equilibrium Systems, University of Nottingham, NG7 2RD, UK \\ ${ }^{2}$ Indian Institute of Science Education and Research, Pune, 411008, India
}

\begin{abstract}
We investigate the dynamics of a three-dimensional Bose-Einstein condensate of ultracold atomic gases with a soft-core shape long-range interaction, which is induced by laser dressing the atoms to a highly excited Rydberg state. For a homogeneous condensate, the long-range interaction drastically alters the dispersion relation of the excitation, supporting both roton and maxon modes. Rotons are typically responsible for the creation of supersolids, while maxons are normally dynamically unstable in BECs with dipolar interactions. We show that maxon modes in the Rydberg-dressed condensate, on the contrary, are dynamically stable. We find that the maxon modes can be excited through an interaction quench, i.e. turning on the soft-core interaction instantaneously. The emergence of the maxon modes is accompanied by oscillations at high frequencies in the quantum depletion, while rotons lead to much slower oscillations. The dynamically stable excitation of the roton and maxon modes leads to persistent oscillations in the quantum depletion. Through a self-consistent Bogoliubov approach, we identify the dependence of the maxon mode on the soft-core interaction. Our study shows that maxon and roton modes can be excited dynamically and simultaneously by quenching Rydberg-dressed long-range interactions. This is relevant to current studies in creating and probing exotic states of matter with ultracold atomic gases.
\end{abstract}

\section{INTRODUCTION}

Collective excitations induced by particle-particle interactions play an important role in the understanding of static and dynamical properties of many-body systems. The ability to routinely create and precisely control properties of ultracold atomic gases opens exciting prospects to manipulate and probe collective excitations. In weakly interacting Bose-Einstein condensates (BECs) with s-wave interactions [1-4], phonon excitations reduce the condensate density, giving rise to quantum depletion [5]. It has been shown [6] that quantum depletion can be enhanced by increasing the s-wave scattering length through Feshbach resonances $[7,8]$. By dynamically changing the s-wave scattering length [9], phonon excitations can alter the quantum depletion, the momentum distribution [10], correlations [11], contact [12, 13], and statistics [14] of the condensate. Moreover the phonon induced quantum depletion plays a vital role in the formation of droplets in BECs [15].

When long-range interactions are introduced, the dispersion relation corresponding to the quasiparticle spectrum of a BEC is qualitatively different, where the excitation energies of the collective modes depend nonmonotonically on the momentum. Previously BECs with dipole-dipole interactions have been extensively examined [16-22]. In two-dimensional (2D) dipolar BECs [23], roton and maxon modes emerge, where roton (maxon) modes correspond to local minima (maxima) in the dispersion relation. The strength of dipolar interactions can be tuned by either external electric or magnetic fields [19]. When instabilities of roton modes are triggered, a homogeneous BEC undergoes density modulations such that a supersolid phase could form. The existence of roton modes has been supported by a recent
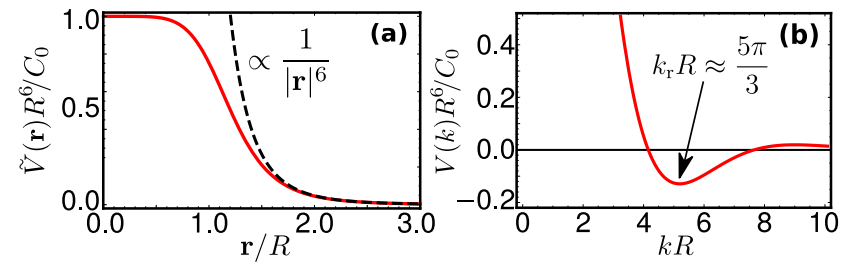

(c)

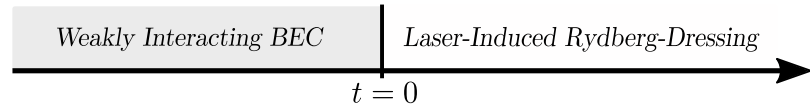

FIG. 1. (color online) Soft-core interaction and quench scheme. (a) The soft-core interaction as a function of the interatomic distance $\mathbf{r}$. Energy is scaled by $R^{6} / C_{0}$ with $R$ and $C_{0}$ to be the soft-core radius and dispersion coefficient. The interaction is constant when $\mathbf{r} \ll R$, and becomes a vdW type when $\mathbf{r} \gg R$. (b) Fourier transformation of the soft-core interaction. The minimum of the interaction locates at $k_{\mathrm{r}} R \approx$ $5 \pi / 3$, where the interaction is attractive. (c) The quench scheme. A weakly interacting BEC with s-wave interactions is first prepared. The laser dressing is applied at $t>0$, which induces the soft-core interaction.

experiment of ultracold dipolar gases [24]. Maxon modes, on the other hand, normally appear at lower momentum states [23]. It was shown however that the maxon modes in dipolar BECs are typically unstable and decay rapidly through the Beliaev damping [20, 21].

Strong and long-range interactions are also found in gases of ultracold Rydberg atoms [25-29]. Rydberg atoms are in highly excited electronic states and interact via long-range van der Waals ( vdW) interactions. The strength of the vdW interaction is proportional to $\mathcal{N}^{11}$ with $\mathcal{N}$ to be the principal quantum number in the Rydberg state. For large $\mathcal{N}$ (current experiments exploit $\mathcal{N}$ typically between 30 and 100), the interaction between 
two Rydberg atoms can be as large as several $\mathrm{MHz}$ at a separation of several micrometers [30]. However lifetimes in Rydberg states are typically $10 \sim 100 \mu \mathrm{s}$, which is not long enough to explore spatial coherence. As a result, Rydberg-dressing, in which a far detuned laser couples electronic ground states to Rydberg states, is proposed. The laser coupling generates a long-range, soft-core type interaction between Rydberg-dressed atoms [31-41]. The coherence time and interaction strength can be controlled by the dressing laser [35]. With this dressed interaction, interesting physics, such as magnets [42], transport [43], supersolids [31, 34, 44, 45], etc, have been studied. Signatures of the dressed interaction have been experimentally demonstrated with atoms trapped in optical lattices and optical tweezers $[42,46]$.

In this paper, we study excitations of roton and maxon modes in three dimensional (3D) Rydberg-dressed BECs in free space at zero temperature. Three dimensional uniform trapping potential of ultracold atoms have been realized experimentally [47]. When the soft-core interaction is strong, both the roton and maxon modes are found in the dispersion relation of the collective excitations. Starting from a weakly interacting BEC, roton and maxon modes are dynamically excited by instantaneously switching on the Rydberg-dressed interaction. Through a self-consistent Bogoliubov calculation, we show that the roton and maxon modes lead to non-equilibrium dynamics, where the quantum depletion exhibits slow and fast oscillations. Through analyzing the Bogoliubov spectra, we identify that the slow oscillations correspond to the excitation of the roton modes, while the fast oscillations come from the excitation of the maxon modes. The dependence these modes have on the quantum depletion in the long time limit is determined analytically and numerically.

The paper is organized as follows. In Sec. II, the Hamiltonian of the system and properties of the soft-core interaction are introduced. Bogoliubov methods, that are capable to study static as well as dynamics of the excitation, are presented. In Sec. III, dispersion relations are found using the static Bogoliubov calculation, where roton and maxon modes are identified. We then examine the dynamics of the quantum depletion due to the interaction quench. Excitations of the roton and maxon modes are studied using a self-consistent Bogoliubov method. The asymptotic behavior of the BEC at long times is also explored. Finally, in Sec. IV we conclude our work.

\section{HAMILTONIAN AND METHOD}

\section{A. Hamiltonian of the Rydberg-dressed BEC}

We consider a uniform 3D Bose gas of $N$ atoms that interact through both s-wave and soft-core interactions.
The Hamiltonian of the system is given by $(\hbar \equiv 1)$,

$$
\begin{aligned}
\hat{H} & =\int \psi^{\dagger}(\mathbf{r})\left(-\frac{\nabla^{2}}{2 m}-\mu\right) \psi(\mathbf{r}) \mathrm{d} \mathbf{r} \\
& +\frac{1}{2} \int \psi^{\dagger}(\mathbf{r}) \psi^{\dagger}\left(\mathbf{r}^{\prime}\right) \tilde{g}\left(\mathbf{r}-\mathbf{r}^{\prime}\right) \psi(\mathbf{r}) \psi\left(\mathbf{r}^{\prime}\right) \mathrm{d} \mathbf{r} \mathrm{d} \mathbf{r}^{\prime},
\end{aligned}
$$

where $\psi(\mathbf{r})$ is the annihilation operator of the bosonic field, $\mu$ is the chemical potential, $m$ is the mass of a boson, and $\nabla$ is the 3D nabla operator on coordinate $\mathbf{r}=\{x, y, z\}$. The interaction potential is described by $\tilde{g}\left(\mathbf{r}-\mathbf{r}^{\prime}\right)=g_{0} \delta(\mathbf{r})+\tilde{V}\left(\mathbf{r}-\mathbf{r}^{\prime}\right)$, where $g_{0}=4 \pi a_{\mathrm{s}} / m$ is the short-range contact interaction controlled by the swave scattering length $a_{\mathrm{s}}[3] . \tilde{V}\left(\mathbf{r}-\mathbf{r}^{\prime}\right)$ is the long-range soft-core interaction,

$$
\tilde{V}\left(\mathbf{r}-\mathbf{r}^{\prime}\right)=\frac{C_{0}}{R^{6}+\left|\mathbf{r}-\mathbf{r}^{\prime}\right|^{6}},
$$

where $C_{0}$ is the strength of the dressed interaction potential and $R$ is the soft-core radius [35]. Both these parameters can be tuned independently by varying the dressing laser [35]. The interaction potential saturates to a constant, i.e. $\tilde{V}(\mathbf{r}) \rightarrow C_{0} / R^{6}$ when $|\mathbf{r}| \ll R$, and approaches to a vdW type at distances of $|\mathbf{r}| \gg R$, i.e. $\tilde{V}(\mathbf{r}) \rightarrow C_{0} /|\mathbf{r}|^{6}$. An example of the soft-core potential is shown in Fig. 1(a). The Fourier transformation of the soft-core potential is $V(k)=U_{0} f(k)$, where $U_{0}=C_{0} / R^{6}$ determines the strength and $f(k)$ has an analytical form

$$
f(k)=\frac{2 \pi^{2} \mathrm{e}^{-\frac{k R}{2}}}{3 k R}\left[\mathrm{e}^{-\frac{k R}{2}}-2 \sin \left(\frac{\pi}{6}-\frac{\sqrt{3} k R}{2}\right)\right],
$$

which characterizes the momentum dependence of the interaction. Though the interaction is repulsive in real space, i.e. $\tilde{V}(\mathbf{r})>0$, it contains negative regions in momentum space, as shown in Fig. 1(b). The negative part of $V(k)$ appears at momentum around $k R \sim 5 \pi / 3$. Previously, it was shown that the attractive interaction is crucially important to the formation of roton instabilities, as revealed by the Bogoliubov approximation [23].

\section{B. Time-independent Bogoliubov approach}

In momentum space, we expand the field operators using a plane wave basis, $\psi(\mathbf{r})=1 / \sqrt{\Omega} \sum_{\mathbf{k}} \mathrm{e}^{\mathrm{ik} \cdot \mathbf{r}} \hat{a}_{\mathbf{k}}$. The many-body Hamiltonian can be rewritten as

$$
\hat{H}=\sum_{\mathbf{k}}\left(\epsilon_{k}-\mu\right) \hat{a}_{\mathbf{k}}^{\dagger} \hat{a}_{\mathbf{k}}+\sum_{\mathbf{q}, \mathbf{k}, \mathbf{k}^{\prime}} \frac{g_{k}}{2 \Omega} \hat{a}_{\mathbf{k}+\mathbf{q}}^{\dagger} \hat{a}_{\mathbf{k}^{\prime}-\mathbf{q}}^{\dagger} \hat{a}_{\mathbf{k}} \hat{a}_{\mathbf{k}^{\prime}}
$$

where $\hat{a}_{\mathbf{k}}^{\dagger}\left(\hat{a}_{\mathbf{k}}\right)$ is the creation (annihilation) operator of the momentum state $\mathbf{k}$, and $\Omega$ volume of the BEC. The kinetic energy is $\epsilon_{k}=k^{2} / 2 m$ with $k=|\mathbf{k}|$, while the Fourier transformation of the atomic interaction $\tilde{g}\left(\mathbf{r}-\mathbf{r}^{\prime}\right)$ is given by $g_{k}=g_{0}+V(k)$.

For a homogeneous condensate and in the stationary regime, we apply a conventional Bogoliubov approach 
$[48,49]$ to study the excitation spectra. At zero temperature we assume a macroscopic occupation in the condensate, which allows us to replace $\hat{a}_{0} \approx \sqrt{N_{0}}$ with $N_{0}$ being the number of condensed atoms. We then apply a canonical transformation on the bosonic operators of the nonzero momentum states [3], $\hat{a}_{\mathbf{k} \neq 0}=\bar{u}_{k} \hat{b}_{\mathbf{k}}-\bar{v}_{k}^{*} \hat{b}_{-\mathbf{k}}^{\dagger}$ where $b_{\mathbf{k}}$ $\left(\hat{b}_{-\mathbf{k}}^{\dagger}\right)$ is the annihilation (creation) operator for bosonic quasiparticles and $\bar{u}_{k}$ and $\bar{v}_{k}$ are complex numbers such that $\left|\bar{u}_{k}\right|^{2}-\left|\bar{v}_{k}\right|^{2}=1$, which satisfies the bosonic commutation relation [3]. The excitation spectra of the Bogoliubov modes for different momentum components give the dispersion relation,

$$
\bar{E}_{k}=\sqrt{\epsilon_{k}\left[\epsilon_{k}+2 g_{k} n_{0}\right]},
$$

with $n_{0}=N_{0} / \Omega$ being the density of the condensed atoms. The coefficients in the Bogoliubov transformation are [3]

$$
\begin{aligned}
\bar{u}_{k} & =\sqrt{\frac{1}{2}\left[\frac{\epsilon_{k}+g_{k} n_{0}}{\bar{E}_{k}}+1\right]} \\
\bar{v}_{k} & =-\sqrt{\frac{1}{2}\left[\frac{\epsilon_{k}+g_{k} n_{0}}{\bar{E}_{k}}-1\right]} .
\end{aligned}
$$

The distribution of the non-condensed atoms is given by $n_{k}=\left\langle a_{k}^{\dagger} a_{k}\right\rangle=\left|\bar{v}_{k}\right|^{2}$. Taking into account contributions from all non-condensed components, the quantum depletion in the stationary state is evaluated as $\bar{n}_{\mathrm{d}}=1 / \Omega \sum_{\mathbf{k} \neq 0}\left|\bar{v}_{k}\right|^{2}$.

\section{Self-consistent Bogoliubov approach for the quench dynamics}

The quench of the soft-core interaction consists of two steps. The system is initially in the ground state of a weakly interacting BEC, i.e. $U_{0}=0$ when $t \leq 0$. At time $t>0$ the Rydberg dressing is switched on immediately. The scheme is depicted in Fig. 1(c). The time dependence of the atomic interaction is described by a piecewise function as follows,

$$
g_{k}= \begin{cases}g_{0} & \text { when } t \leq 0 \\ g_{0}+U_{0} f(k) & \text { when } t>0 .\end{cases}
$$

We assume that the s-wave interaction is not affected during the quench. Hence we use parameter $\alpha=U_{0} / g_{0}$ to characterize the strength of the soft-core interaction with respect to the s-wave interaction.

A time-dependent Bogoliubov approach is applied to study the dynamics induced by the interaction quench. It is an extension of the conventional Bogoliubov approximation, where the canonical transformation becomes time-dependent, $\hat{a}_{\mathbf{k} \neq 0}(t)=u_{k}(t) \hat{b}_{\mathbf{k}}-v_{k}(t)^{*} \hat{b}_{-\mathbf{k}}^{\dagger}$ where $u_{k}(t)$ and $v_{k}(t)$ are time-dependent amplitudes with the relation $\left|u_{k}(t)\right|^{2}-\left|v_{k}(t)\right|^{2}=1$, which preserves the bosonic commutation relation. This approach has been widely used to study excitation dynamics in BECs with or without long-range interactions $[10-12,14,20]$. It provides a good approximation when the condensate has not undergone significant depletion.

Using the Heisenberg equation of the bosonic operators, we obtain equations of motion of $u_{k}(t)$ and $v_{k}(t)$,

$$
i\left[\begin{array}{c}
\dot{u}_{k}(t) \\
\dot{v}_{k}(t)
\end{array}\right]=\left[\begin{array}{cc}
\epsilon_{k}+g_{k} n_{\mathrm{c}}(t) & g_{k} n_{\mathrm{c}}(t) \\
-g_{k} n_{\mathrm{c}}(t) & -\epsilon_{k}-g_{k} n_{\mathrm{c}}(t)
\end{array}\right]\left[\begin{array}{l}
u_{k}(t) \\
v_{k}(t)
\end{array}\right],
$$

where $n_{\mathrm{c}}(t)$ is the condensate density. The total density consists of the condensate and depletion densities as $n=$ $n_{\mathrm{c}}(t)+n_{\mathrm{d}}(t)$ with the total density of the excitation, i.e. quantum depletion given as

$$
n_{\mathrm{d}}(t)=\frac{1}{\Omega} \sum_{k} n_{k}(t)=\frac{1}{2 \pi^{2}} \int_{0}^{\infty} n_{k}(t) k^{2} \mathrm{~d} k,
$$

where $n_{k}(t) \equiv\left\langle\hat{a}_{\mathbf{k}}^{\dagger} \hat{a}_{\mathbf{k}}\right\rangle=\left|v_{\mathbf{k}}(t)\right|^{2}$ is the distribution of momentum states. When the excitation from the condensate is weak $n_{\mathrm{d}} \approx 0$, we can approximate $n_{\mathrm{c}}(t) \approx n$. Eqs. (7) can be solved exactly,

$$
\begin{aligned}
{\left[\begin{array}{l}
u_{k}(t) \\
v_{k}(t)
\end{array}\right] } & =\left[\cos \left(E_{k}(t) t\right) \mathbb{I}-\mathrm{i} \frac{\sin \left(E_{k}(t) t\right)}{E_{k}(t)}\right. \\
& \left.\times\left(\begin{array}{cc}
\epsilon_{k}+g_{k} n_{\mathrm{c}}(t) & g_{k} n_{\mathrm{c}}(t) \\
-g_{k} n_{\mathrm{c}}(t) & -\epsilon_{k}-g_{k} n_{\mathrm{c}}(t)
\end{array}\right)\right]\left[\begin{array}{l}
u_{k}(0) \\
v_{k}(0)
\end{array}\right]
\end{aligned}
$$

where $\mathbb{I}$ is the identity matrix, and the dispersion relation $E_{k}(t)=\sqrt{\epsilon_{k}\left[\epsilon_{k}+2 g_{k} n_{\mathrm{c}}(t)\right]}$. The initial values of $u_{k}(t)$ and $v_{k}(t)$ are [3],

$$
\begin{aligned}
& u_{k}(0)=\sqrt{\frac{1}{2}\left[\frac{\epsilon_{k}+g_{0} n}{E_{k}(0)}+1\right]} \\
& v_{k}(0)=-\sqrt{\frac{1}{2}\left[\frac{\epsilon_{k}+g_{0} n}{E_{k}(0)}-1\right]} .
\end{aligned}
$$

We kept $n_{\mathrm{c}}(t)$ explicitly in Eq. (9) to indicate that $u_{k}(t)$ and $v_{k}(t)$ are time-dependent quantities, while the total density $n$ is time-independent. Using the time-dependent solutions, we calculate the momentum distribution $n_{k}(t)$,

$$
\begin{aligned}
n_{k}(t)= & \left|v_{k}(0)\right|^{2}+g_{k} n_{\mathrm{c}}(t)\left[g_{k} n_{\mathrm{c}}(t)-g_{0} n\right] \\
& \times \frac{\epsilon_{k}\left[1-\cos \left(2 E_{k}(t) t\right)\right]}{2 E_{k}(t)^{2} E_{k}(0)} .
\end{aligned}
$$

Eq. (11) shows that $n_{k}(t)$ encodes the dispersion relation. One can see this after carrying out a Fourier transform of $n_{k}(t)$ to the frequency domain [50].

As we will consider very weak quantum depletion, the conventional Bogoliubov approach can already describe the dynamics well. To take into account the corrections due to the weak quantum depletion, we additionally employ a self-consistent method through iterative calculations. From Eq. (9), we obtain the quantum depletion and hence $n_{\mathrm{c}}(t)$. As we still have $n_{\mathrm{c}}(t) \approx n, n_{\mathrm{c}}(t)$ will be treated as an adiabatically changing quantity such that we can again use Eq. (9) to calculate the quantum depletion in which the weak time-dependence of $n_{\mathrm{c}}(t)$ will be 
considered. This procedure will be iterated until $n_{\mathrm{c}}(t)$ is self-consistently obtained, i.e. additional iterations will not change $n_{\mathrm{c}}(t)$ any more. Note that the procedure used here is approximately applicable as the quantum depletion is small. When the depletion is strong, one could apply the rigorous self-consistent procedure presented in Ref. [12].

In the following calculations, we will scale the energies, lengths, and times with respect to the interaction energy $g_{0} n$, coherence length $\zeta=\left(m g_{0} n\right)^{-1 / 2}$, and coherence time $\tau=t g_{0} n$ of the initial condensate. The zero range interaction strength is fixed by the s-wave scattering length. To be concrete we will set $a_{\mathrm{s}}=0.1 n^{-1 / 3}$ throughout this work.

\section{RESULTS AND DISCUSSIONS}

\section{A. Stationary dispersion relation}

The soft-core interaction drastically alters the dispersion relation of the Bogoliubov excitations. To illustrate this, we first examine dispersion relations of a static BEC by assuming that the soft-core interaction is present. When the soft-core interaction is weak, i.e. $\alpha$ is small, the dispersion relation resembles that of a weakly interacting BEC. The excitation energies increase monotonically with momentum $k$ [3] [see Fig. 2(a)]. By increasing $\alpha$, the shape of the Bogoliubov spectra changes significantly. For different $\alpha$, the dispersion intersects at a momentum determined by $V(k)=0$ [see Fig. 1(b)], where mode energies of the Rydberg-dressed BEC coincide with that of a weakly interacting BEC (the dashed curve). More importantly a local maximum and minimum can be seen in the dispersion relation when $\alpha$ is large [Fig. 2(a)]. At the maximum, special modes called maxon modes form, while roton modes emerge around the minima [23]. In the following, we will denote the energies of the maxons and rotons with $\gamma_{\mathrm{m}}$ and $\gamma_{\mathrm{r}}$, as the local maximal and minimal values of the dispersion relation.

The roton and maxon modes depend on the soft-core interaction non-trivially. When increasing $\alpha, \gamma_{\mathrm{r}}$ decreases while $\gamma_{\mathrm{m}}$ increases, as given by the examples shown in Fig. 2(a). For sufficiently large $\alpha$, the roton gap vanishes as the energies become complex. Due to the roton instability, the homogeneous state becomes dynamically unstable which leads to interesting physics. It has been shown that the emergence of the roton instability can cause a first order phase transition where the ground state changes from a uniform condensate to a supersolid state $[35,51,52]$. We note that instabilities in dipolar BECs are caused by angular dependent interactions with both attractive and repulsive components [16], while the instability in the dressed BEC is induced by stronger, isotropic interactions. We will show in the following section that switching on the dressed interaction induces exotic dynamics even without triggering the roton instability.
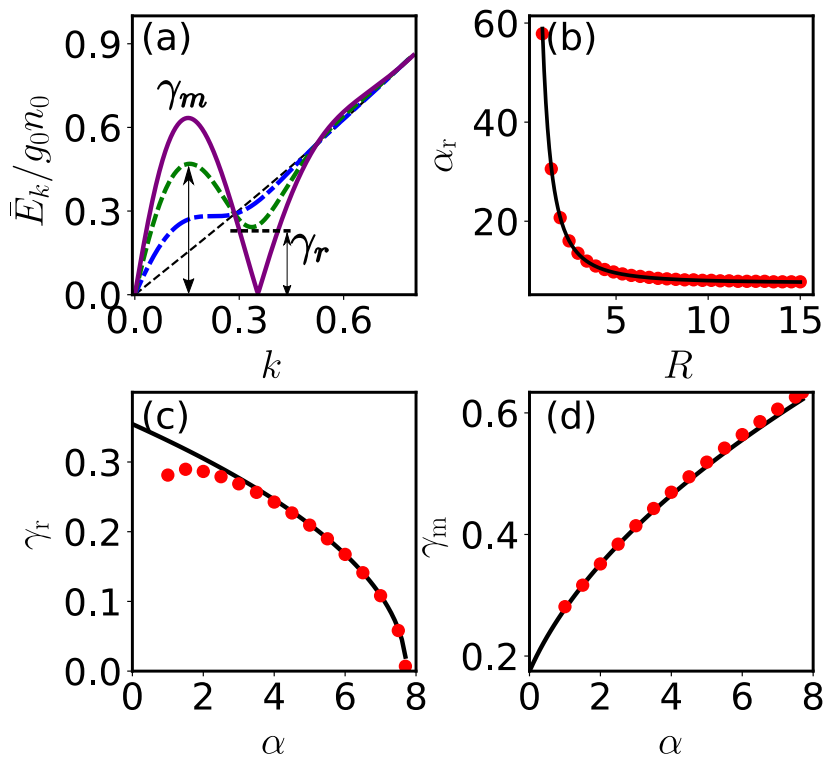

FIG. 2. (color online) Roton and maxon modes. (a) Bogoliubov spectra in the stationary state for $\alpha=0$ (black, thin dashed), 1 (blue, dotdashed), 6 (green, thick dashed), and 7.7 (purple, solid), when $R=15$. The energy gaps $\gamma_{\mathrm{r}}$ and $\gamma_{\mathrm{m}}$ indicating respectively the roton and maxon energies are marked for the green curve. For $\alpha>7.7$, the spectra becomes unstable. (b) The critical value $\alpha_{\mathrm{r}}$ vs $R$. Analytical calculations (black) agree with the numerical data (red dots). (c) Roton energy $\gamma_{\mathrm{r}}$. Increasing $\alpha$, the roton energy decreases. For large $\alpha$, the analytical (black solid) and numerical (dot) results agree. At small $\alpha$, roton minima become weak and eventually disappear, which leads to the deviation. The data points in red are the energies taken numerically from the dispersion. (d) Maxon energy $\gamma_{\mathrm{m}}$ increases with $\alpha$. The analytical (black solid) and numerical data agree nicely. In (c) and (d) $R=15$.

We now obtain the critical value at which the roton mode becomes unstable. From Fig. 1(b), the Fourier transform of the soft-core potential has the most negative value around $k_{\mathrm{r}} \approx 5 \pi / 3 R$. The roton minimum takes place around this momentum. By substituting $k_{\mathrm{r}}$ into the dispersion relation, we can identify the critical $\alpha$ at which the roton energy becomes complex,

$$
\alpha_{\mathrm{r}}=\frac{5 \mathrm{e}^{5 \pi / 3}\left(36 R^{2}+25 \pi^{2}\right)}{72 \pi R^{2}\left[2 \mathrm{e}^{5 \pi / 6} \sin \left(\frac{\pi}{6}-\frac{5 \pi}{2 \sqrt{3}}\right)-1\right]} .
$$

To check the accuracy of this critical value, we numerically find the instability point from the dispersion relation for various $\alpha$ values. Both numerical and analytical values are shown in Fig. 2(b). The analytical result agrees with the numerical values very well. This supports the assumption that the roton minimum happens around momentum $k_{\mathrm{r}}$.

Knowing the momentum $k_{\mathrm{r}}$, we can obtain the roton energies by inserting it into Eq. (4). It is found that 

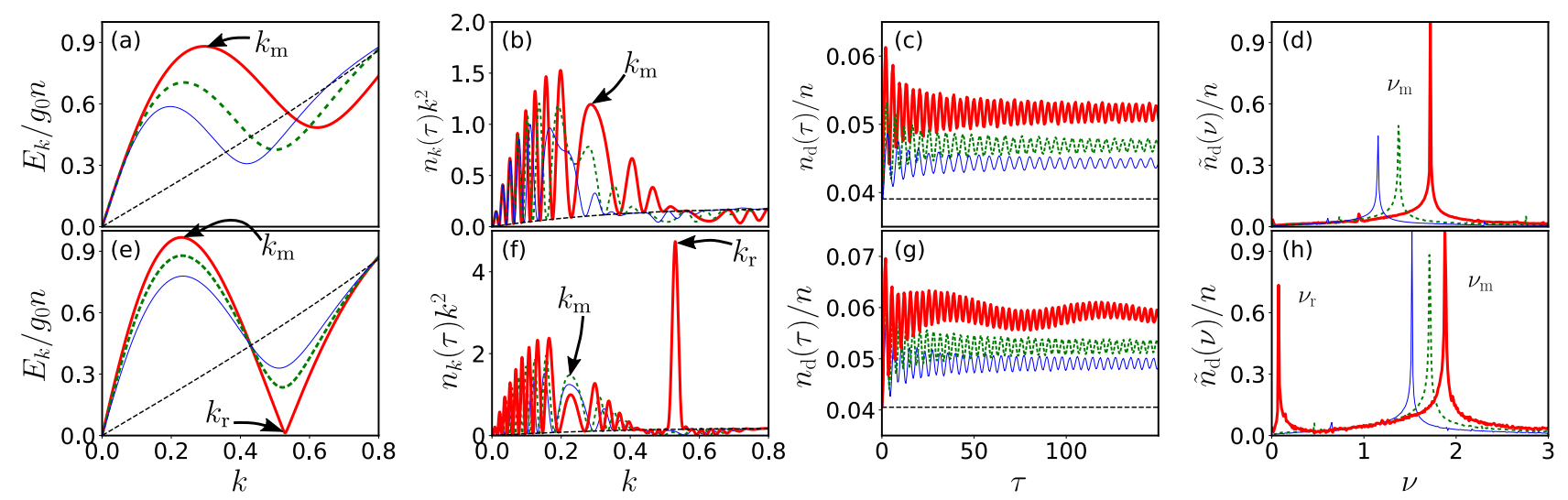

FIG. 3. (color online) Excitation of the roton and maxon mode. In the upper panels, (a) gives the dispersion for a static BEC. The momentum of the roton and maxon modes decreases with increasing soft-core radius $R$. Without soft-core interactions, the excitation energy monotonically increases with momentum (black, thin dashes). The location of the maxon modes for the red curve is highlighted it the arrow. In (b)-(c), the interaction quench is applied. Momentum densities $n_{k} k^{2}$ at time $\tau=30$ are shown in (b). The black dashed curve shows the momentum distribution of the initial state. The quantum depletion damps slightly at early times and then oscillate rapidly with negligible damping over a long time (c). This leads to sharp peaks in the respective Fourier transformation (d). The non-zero width of the peaks results from the damping at the early stage of the evolution. The frequency $\nu_{\mathrm{m}}$ at the major peaks is determined by the maxon frequency. Minor peaks corresponding to other frequencies are almost invisible. In (a)-(d), three different soft-core radius $R=8$ (red, thick), 10 (green, thick dashes), and 12 (blue, thin) are considered, while the interaction strength is fixed at $\alpha=4$. In the lower panels, the dispersion (e), momentum distribution (f), quantum depletion (g) and Fourier transformation of the quantum depletion (h) for $R=10$ and $\alpha=5$ (blue, thin), 6.5 (green, thick dashes), and 7.99 (red, thick) are shown. Approaching to the roton instability (e), the momentum density distribution (f) develops a large occupation around modes at $k_{\mathrm{r}}$ at $\tau=30$. Both the roton and maxon momenta are highlighted with arrows in this case. The depletion dynamics maintains a slower oscillation (g) as the interaction strength is increased, which can be seen from the Fourier transformation of the quantum depletion (h). The lower peak frequency $\nu_{\mathrm{r}}$ is determined by the roton mode. The major peaks at higher frequencies are due to the excitation of maxons. When $\alpha=7.99$, both the roton and maxon mode are dynamically stable, giving narrow Fourier spectra. While calculating the above spectra, the system was allowed to evolve up to time $\tau=600$.

the roton energy $\gamma_{\mathrm{r}}$ decreases with increasing $\alpha$ [see Fig. $2(\mathrm{c})]$. The roton energy from the numerical calculations agrees with the analytical data, especially when the softcore interaction is strong. Decreasing the soft-core interaction, the roton modes disappear for sufficiently small $\alpha$, as our numerical calculations indicate. Here large deviations between the two methods are found in this regime.

On the other hand, the location of the maxon modes in momentum space is difficult to find. By analyzing the dispersion relation, the momentum corresponding to the maxon mode is approximately given by $k_{\mathrm{m}} \approx k_{\mathrm{r}} / 2$. Using this approximation, we substitute this momentum value into Eq. (4) and calculate the maxon energy. The result is shown in Fig. 2(d), where the approximate value matches the numerical values with a high degree of accuracy.

Recently, the stationary state of 2D and 3D Rydbergdressed BECs have been examined [53]. It was shown that the increased occupation around the roton modes leads to instabilities in the ground state in the form of density waves. It was also seen that the strong interparticle interactions lead to a large depletion of the condensate.

\section{B. Roton and maxon excitation}

Depending on parameters of the soft-core interaction, the stationary dispersion relation could support roton and maxon modes. One example is displayed in Fig. $3(\mathrm{a})$. Now if we quench the interaction, the dispersion relations of the initial and final states are different. The system is driven out of equilibrium, such that the momentum distributions $n_{k}(\tau)$ evolve with time. In Fig 3(b), snapshots of the momentum density $n_{k}(\tau) k^{2}$ are shown. At $\tau=0$, the BEC is in a stationary state, which depends on the initial condition, $\bar{v}_{k}$. The respective momentum density is a smooth function of $k$. At later times, different momentum components are excited by the presence of the soft-core interaction causes dynamical evolution of the quantum depletion.

The dynamics of the quantum depletion depends vitally on the parameter $R$ and $\alpha$ in the soft-core interaction. After switching on the interaction, the excitation of the Bogoliubov modes significantly affects the momentum distribution. We will first investigate the oscillatory behavior of the quantum depletion. For moderate soft-core interactions, many momentum modes are excited by the soft-core interaction, as shown in Fig. 3(b). 
The quantum depletion increases rapidly at short times and then oscillates around a constant value [Fig. 3(c)]. Its amplitude decreases slowly when $\tau<100$ but then reaches a constant. The Fourier transformation $\tilde{n}_{\mathrm{d}}(\nu)$ of the quantum depletion, characterizing the spectra of the dynamics, shows a sharp peak [Fig. 3(d)]. The finite width of the peak is largely due to the damping of the quantum depletion at the early stage of the evolution. The peak positions, i.e. frequency of the oscillations, decrease gradually when increasing the soft-core radius.

For stronger soft-core interactions, the roton mode moves towards the instability point [see Fig. 3(e)]. As the interaction strength approaches $\alpha_{\mathrm{r}}$, the momentum density develops a large occupation at momentum values matching $k_{\mathrm{r}}$ [see Fig. 3(f)]. Around the maxon momentum $k_{\mathrm{m}}$, there are also large occupations. A new, lower frequency pattern develops on top of the fast oscillation in the quantum depletion [Fig. $3(\mathrm{~g})]$. This changes the Fourier spectra of the quantum depletion, where a new peak is found at a lower frequency [Fig. 3(h)].

Importantly, the peak positions in $\tilde{n}_{\mathrm{d}}(\nu)$ are determined by the roton and maxon energies, where the fast oscillations are due to the excitations of the maxon modes, while slow oscillations are due to the roton modes. To verify this, we first obtain the maxon and roton frequencies by substituting the corresponding momentum $k_{\mathrm{m}}$ and $k_{\mathrm{r}}$ into Eq. (4). We then compare them with the frequency at the peak positions in the Fourier spectra. Note that the oscillation frequency (i.e. peak frequency of the Fourier spectra) in the quantum depletion is twice the Bogoliubov energy, as can be seen in Eq. (11). As shown in Fig. 4, the numerical data for both the maxon mode (a-b) and roton mode (c-d) agree with the analytical calculations. When varying the interaction strength, the maxon (roton) frequency increases (decreases) with increasing $\alpha$. If we increase the soft-core radius, frequencies of both modes decrease.

The agreement between numerical and analytical calculation confirm that both roton and maxon modes are excited via quenching the soft-core interaction. The dynamically excited modes are stable, as both the fast and slow oscillations are persistent for a long time. In our numerical simulations, the oscillations will not dampen even when the simulation time $\tau>1000$. Such persistent oscillatory dynamics also leads to the sharp peaks in the Fourier transformation of the quantum depletion.

We want to emphasize that the quench dynamics in the dressed BEC is in sharp contrast to BECs with either s-wave or dipolar interactions. In a weakly interacting BEC, the quantum depletion grows exponentially to a steady value $\propto \zeta^{-\frac{1}{3}}$, while oscillatory patterns are not present in the depletion [11], due to the fact that low energy phonon modes dominate the quench dynamics. In dipolar BECs [19, 20, 24, 54, 55], on the other hand, roton modes are formed due to the interplay between long-range dipolar and s-wave interactions $[19,20,24$, $54,55]$. These roton modes can be excited by quenching the dipolar interaction, while maxon modes are typically
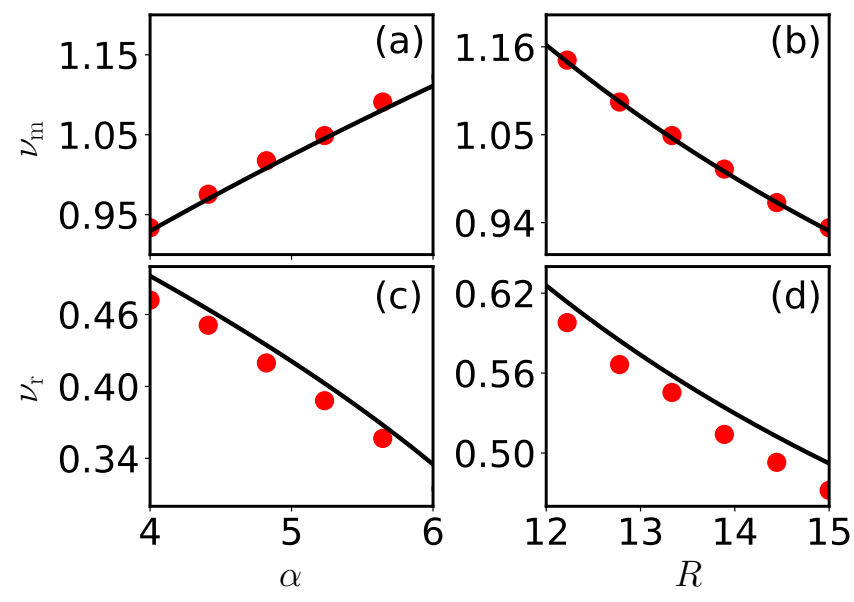

FIG. 4. (color online) Maxon frequency (a-b) and roton frequency (c-d). The dots are numerical data from the Fourier spectra. The solid curves are the analytical results $2 \gamma_{\mathrm{m}}$ in (a)-(b) and $2 \gamma_{\mathrm{r}}$ in (c)-(d) obtained from the Bogoliubov dispersion. The maxon (roton) frequency increases (decreases) with increasing interaction strength. At the critical point $\alpha_{\mathrm{r}}$, the roton mode loses stability. Frequencies of both modes tends towards 0 for larger $R$ values as the soft-core interaction becomes weaker. In (a) and (c) $R=15$. In (b) and (d) $\alpha=4$.

unstable in the dynamics [see Appendix A for examples].

\section{Quantum depletion in the long time limit}

In the long time limit $\tau \gg 1$, the quantum depletion oscillates rapidly around a mean value [Fig. 3(c) and (g)]. In the following, we will evaluate the asymptotic value of the quantum depletion. First we will derive an analytic expression using the following approximations. In the long time limit, the time averaged quantum depletion is largely determined by the low momentum modes. Moreover, we will neglect the oscillation term in Eq. (11), as they are related to the roton and maxons. Using these approximations, the asymptotic form of the momentum distribution $n_{k}^{\infty}$ is obtained,

$$
n_{k}^{\infty} \approx \frac{1}{2}\left(\frac{k^{2}+1}{\sqrt{k^{2}\left(k^{2}+4\right)}}-1\right)+\frac{\alpha f(k)}{4 k} \frac{n_{\mathrm{c}}^{\infty}}{n},
$$

where $n_{\mathrm{c}}^{\infty}$ is the asymptotic condensate density. After carrying out the integral over momentum space, the approximate quantum depletion when $\tau \rightarrow \infty$ is obtained,

$$
\frac{n_{\mathrm{d}}^{\infty}}{n} \approx 2 \Gamma\left(\frac{R^{2}+\alpha \pi}{3 R^{2}+2 \pi \alpha \Gamma}\right)
$$

where $\Gamma=\left(2 \pi^{2} \zeta^{3} n\right)^{-1}$. This result predicts that the quantum depletion approaches to a constant value $n_{\mathrm{d}}^{\infty} / n \rightarrow 2 \Gamma / 3$ in the limit $R \rightarrow \infty$. This resembles the 

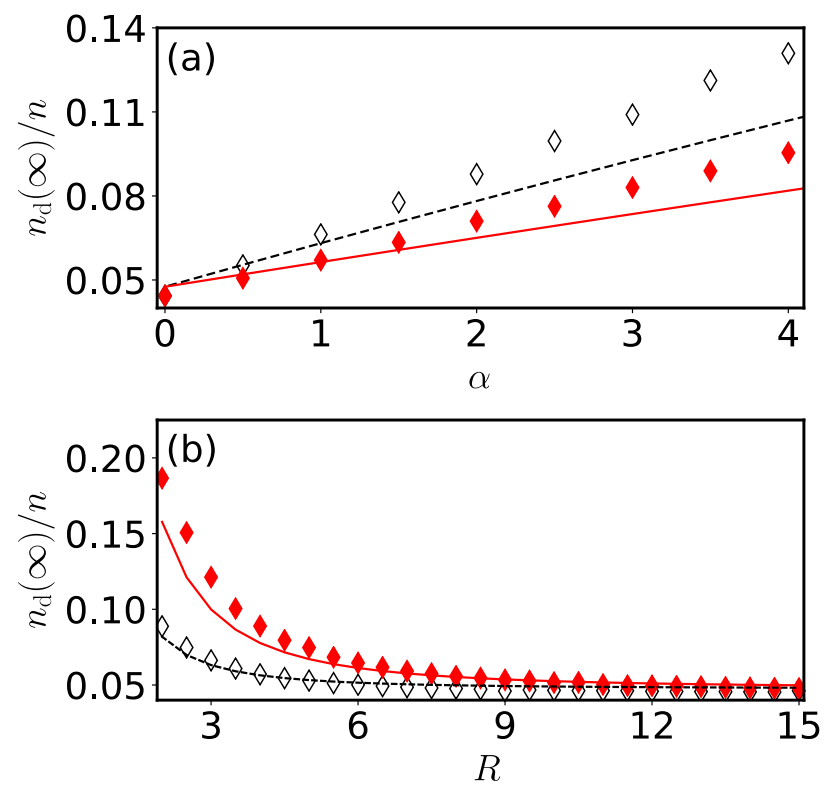

FIG. 5. (color online) Asymptotic quantum depletion. The asymptotic quantum depletion increases with increasing $\alpha$ (a), which is seen from both the analytical and numerical calculations. The quantum depletion $n_{\mathrm{d}}^{\infty}$ decreases with increasing soft-core radius (b). The lines are found analytically using Eq. (14), while the data points are found by numerically solving Eq. (8) and taking the mean value at later times between $\tau \approx 50 \rightarrow 150$. Parameters in (a) are $R=3$ (black, open diamonds, with dashed line) and 4 (red, filled diamond, with solid line). Parameters in (b) are $\alpha=1$ (black, open diamonds, with dashed line) and 3.5 (red, filled diamond, with solid line).

result of the weakly interacting BEC, i.e. the soft-core interactions plays no role in the dynamics.

To verify the analytical calculation, we numerically find the mean value of the quantum depletion when time is large. Both the numerical and analytical results are shown in Fig. 5. For small $\alpha$, low momentum states are populated by switching on the soft-core interaction. This is the regime where the approximation works. Here we find a good agreement between the numerical and analytical calculations. Increasing the interaction strength, more and more higher momentum components are populated [see Fig. 3(f)], causing larger depletion. When fixing $\alpha$, the quantum depletion decreases with increasing $R$, as can be see in Fig. 5(b). This results from the fact that the quench only affects momentum components $k<k_{\mathrm{r}} / R$ [see Fig. 1(b)]. For momentum $k \gg k_{\mathrm{r}} / R$, the dispersion is largely unaffected by the soft-core interaction as the respective $V(k) \rightarrow 0$. Therefore increasing $R$ leads to a weaker quantum depletion.

\section{CONCLUSION}

We have studied dynamics of 3D BECs in free space, with Rydberg-dressed soft-core interactions. An interaction quench is implemented through turning on the softcore interaction instantaneously, starting from a weakly interacting BEC. The Bogoliubov spectra of the BEC displays local maxima and minima, which are identified as maxon and roton modes. Through a time-dependent Bogoliubov approach, we have calculated dynamics of the quantum depletion self-consistently. Our results show that both roton and maxon modes are excited by switching on the soft-core interaction. The excitation of roton and maxon modes generate slow and fast oscillatory dynamics in the quantum depletion. Our simulations show that the excited roton and maxon modes are stable in the presence of the soft-core interaction, which are observed from the persistent oscillations of the quantum depletion. We have found the frequencies of the roton and maxon modes approximately, which are confirmed by the numerical simulations.

Our study shows that exotic roton and maxon excitations can be created in Rydberg-dressed BECs through interaction quenches. Properties of the maxons and rotons can also been seen from condensate fluctuations [see Appendix B for details] and density-density correlations [see Appendix C]. The results studied in this work might be useful in identifying the soft-core interaction, through measuring the frequencies and strength of the quantum depletion. In the future, it is worth studying the stability of the maxon mode due to the Beliav damping $[56,57]$. Maxon modes are also present in strongly correlated ${ }^{4} \mathrm{He}[58,59]$, where the maxon decays into phonons at the Pitaevskii plateau. Whether the maxons in Rydbergdressed BECs will decay through this mechanism is an open and interesting research question. Another interesting question is whether the soft-core interaction can lead to the formation of droplets in Rydberg-dressed BECs.

\section{ACKNOWLEDGEMENTS}

We thank Yijia Zhou and S Kumar Mallavarapu for fruitful discussions. The research leading to these results has received funding from the EPSRC Grant No. EP/M014266/1, the EPSRC Grant No. EP/R04340X/1 via the QuantERA project "ERyQSenS", the UKIERIUGC Thematic Partnership No. IND/CONT/G/16$17 / 73$, and the Royal Society through the International Exchanges Cost Share award No. IEC $\backslash$ NSFC $\backslash 181078$. We are grateful for access to the Augusta High Performance Computing Facility in the University of Nottingham. 


\section{Appendix A: Dynamics of 2D Dipolar Systems}

Quench dynamics in BECs with dipolar interactions are drastically different. The dipolar interaction is given by

$$
\tilde{V}_{\mathrm{dd}}\left(\mathbf{r}-\mathbf{r}^{\prime}\right)=g_{0} \delta(\mathbf{r})+\frac{d^{2}}{\left|\mathbf{r}-\mathbf{r}^{\prime}\right|^{3}}\left[1-3 \cos ^{2}(\theta)\right],
$$

where $d$ is the dipole moment, $\theta$ is the angle between the dipoles and molecular axis, and $g_{0}$ is the short-range contact interaction as before. In 3D, the Fourier transform of the dipolar interaction has no momentum dependence [60]. In a 2D trapped dipolar Bose gas [17, 18], the interaction potential displays a strong momentum dependence [20].

We consider a quasi-2D setup [20], where a strong confinement is applied in the perpendicular $z$-direction while leaving atoms free to move in the $x-y$ plane. The dipoles are polarized along this $z$-axis. This leads the axial confinement as $l_{z}$, which provides a natural rescaling of $\mathbf{r} \mapsto \mathbf{r} / l_{z}[17,18,20-22]$. After integrating Eq. (A1) in the $z$-axis, we obtain the Fourier transformation of the quasi-2D dipolar interaction [20]

$$
g_{\mathrm{dd}}(k)=g_{0}+d^{2}\left[2-3 k \sqrt{\pi} \operatorname{Erfc}(k) \mathrm{e}^{k^{2}}\right],
$$

where $\operatorname{Erfc}(k)$ is the complimentary error function. Here we define the dimensionless parameter $\alpha_{\mathrm{d}}=d^{2} / g_{0}$ to characterizing the strength of the dipolar interaction, such that the interaction after the quench is given as $g_{\text {dd }}(k) / g_{0}=1+\alpha_{\mathrm{d}}\left[2-3 k \sqrt{\pi} \operatorname{Erfc}(k) \mathrm{e}^{k^{2}}\right]$. The quench scheme for the dipolar case is similar to the procedure outlined in the main text. We switch on the dipolar interaction instantaneously, while keeping the s-wave interaction unchanged.

The dispersion relation for the dipolar BEC is shown in Fig. A1(a), where both roton and maxon modes can be seen.

When the dipolar interaction is compared to the Rydberg-dressed BEC [e.g Fig. 2(a)], the energies of the low momentum modes remain small, as seen by directly comparing the dispersion relations. The absence of these large maxon energies means that the mechanism behind the dipolar interactions prevent the oscillations that we previously attributed to the maxon modes from reaching large amplitudes [Fig. A1(b)][20, 21, 61].

The energy spectrum of dipolar BECs [Fig. A1(a)] shows that the maxon energies are slightly above the energy of a weakly interacting BEC (black, dashed line). In comparison, Fig. 2(a) in the main text shows that the maxon energy in the Rydberg-dressed interaction is much higher. The absence of the high frequency oscillations in the dipolar BECs might be attributed to this lack of the high energy maxon modes.

We follow the same self-consistent process to obtain the condensate fraction. We calculate the quantum depletion as before as $n_{\mathrm{d}} / n=1 /\left(2 \pi l_{z}^{2} n\right) \int_{0}^{\infty} n_{k} k \mathrm{~d} k$. When
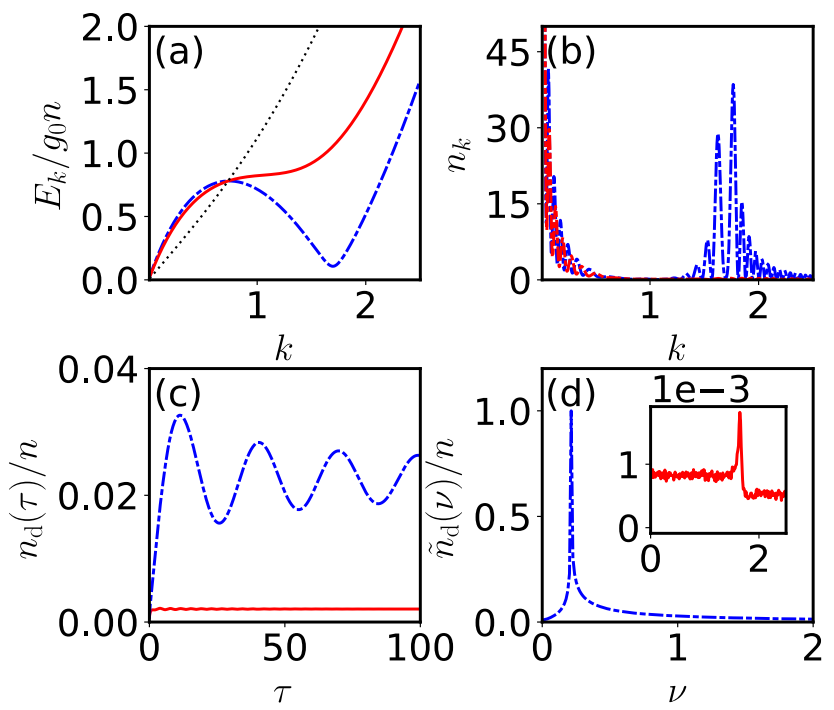

FIG. A1. (color online) Quantum depletion in a dipolar BEC. Solid red curves are for $\alpha_{\mathrm{d}}=2.1$ and blue dashed curves are for $\alpha_{\mathrm{d}}=2.7$. The black dashes correspond to the noninteracting dispersion relation. The axial confinement is set to $l_{z}=0.1 n^{-1 / 2}$. We show the dispersion relation in (a) while the momentum distribution at time $\tau=30$ is shown for (b). The quantum depletion and corresponding Fourier spectra are shown in (c) and (d) respectively. The inset shows a maxon mode is excited for $\alpha_{\mathrm{d}}=2.1$. However the signal is very weak and almost invisible. The axes of the inset is same as panel $(d)$.

$\alpha_{\mathrm{d}}$ is small, the dynamics develops maxon oscillations, which dampens in short time scales, as shown in Fig. $\mathrm{A} 1$ (c). When $\alpha_{\mathrm{d}}$ is large, the roton frequency completely overpowers the maxon frequency in the dynamics. The absence of a stable maxon mode is also seen in the Fourier spectra [Fig. A1(d)].

\section{Appendix B: Condensate fluctuation}

In this section, we evaluate the fluctuation of the condensate for the Rydberg-dressed BEC. The condensate fluctuation is defined as

$$
\begin{aligned}
\Delta n_{\mathrm{c}} & =\sqrt{\left\langle n_{\mathrm{c}}^{2}\right\rangle-\left\langle n_{\mathrm{c}}\right\rangle^{2}} \\
& =\sqrt{\left\langle n_{\mathrm{d}}^{2}\right\rangle-\left\langle n_{\mathrm{d}}\right\rangle^{2}} \\
& =\frac{1}{\Omega} \sqrt{\sum_{\mathbf{k k}^{\prime} \neq 0}\left[\left\langle\hat{a}_{\mathbf{k}}^{\dagger} \hat{a}_{\mathbf{k}} \hat{a}_{\mathbf{k}^{\prime}}^{\dagger} \hat{a}_{\mathbf{k}^{\prime}}\right\rangle-\left\langle\hat{a}_{\mathbf{k}}^{\dagger} \hat{a}_{\mathbf{k}}\right\rangle\left\langle\hat{a}_{\mathbf{k}^{\prime}}^{\dagger} \hat{a}_{\mathbf{k}^{\prime}}\right\rangle\right]},
\end{aligned}
$$

where we have assumed the total density $n$ is a constant. Using the Bogoliubov transformation, the fluctuation of the condensate is obtained,

$$
\Delta n_{\mathrm{c}}=\frac{1}{\Omega} \sqrt{2 \sum_{\mathbf{k} \neq 0} n_{k}\left(1+n_{k}\right)}
$$



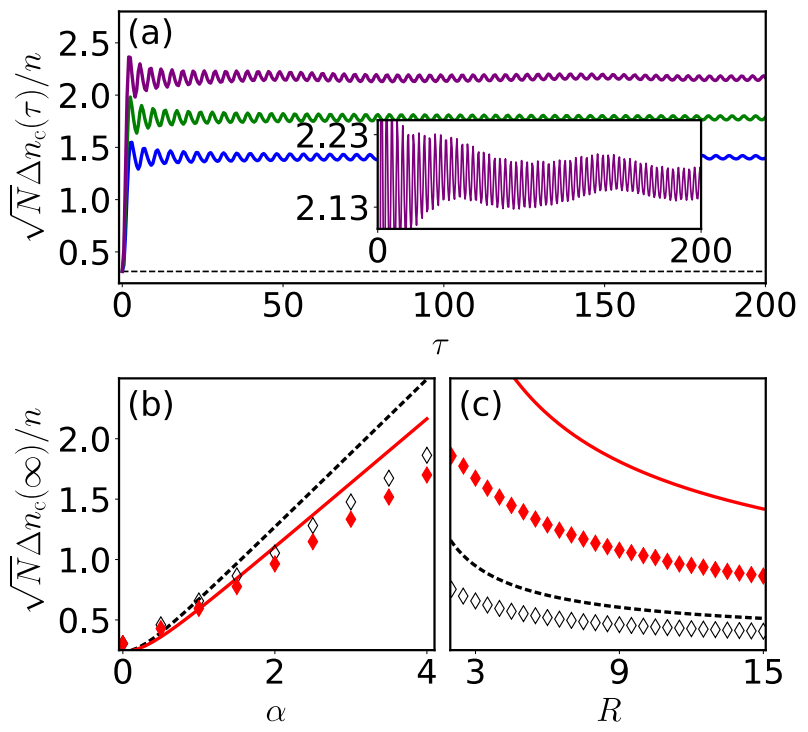

FIG. B1. (color online) Condensate fluctuation. (a) Dynamics of the condensate fluctuation. We fix $R=10$, and evolve the system for $\alpha=5$ (blue, lower curve), 6.5 (green, middle curve), and 7.99 (purple, upper curve). The dashed line is the fluctuation without the soft-core interaction, i.e. $\alpha=0$. The inset shows fluctuations when $\alpha=7.99$ to highlight the low frequency oscillations due to rotons. The axes of the inset is same as panel (a). Mean values of the fluctuations for different $\alpha$ (b) and $R$ (c) when time $\tau \rightarrow \infty$ are shown. We have considered $R=3$ (black, open diamonds, with dashed line) and 4 (red, filled diamond, with solid line) in (b) and $\alpha=1$ (black, open diamonds, with dashed line) and 3.5 (red, filled diamond, with solid line) in (c). Diamond points correspond to the numerical data, while lines represent the analytical expression. Other parameters can be found in Fig. 5 in the main text.

One can numerically evaluate the fluctuation by inserting Eq. (11) into the above equation. For convenience, the relative fluctuation, $\sqrt{N} \Delta n_{\mathrm{c}} / n$, will be calculated. Some examples are shown in Fig. B1(a). The fluctuation increases rapidly, and then saturates at an asymptotic value when time is large. The fluctuation oscillates around the asymptotic value. The maxon modes lead to fast oscillations. When the roton mode is significantly populated, a slower oscillation is found.

The asymptotic value of the fluctuation depends on the soft-core interaction. Increasing $\alpha$, the asymptotic value increases [see Fig. B1(a) and (b)]. We can estimate the asymptotic value of the density fluctuation by replacing $n_{k}$ with its asymptotic value Eq. (13), in Eq. (B1), which yields

$$
\frac{\sqrt{N} \Delta n_{\mathrm{c}}^{\infty}}{n}=\sqrt{2 \Gamma \int_{0}^{\infty} n_{k}^{\infty}\left[1+n_{k}^{\infty}\right] k^{2} \mathrm{~d} k .}
$$

Further assuming the fluctuation depends solely on low momentum states, we obtain the approximate result of
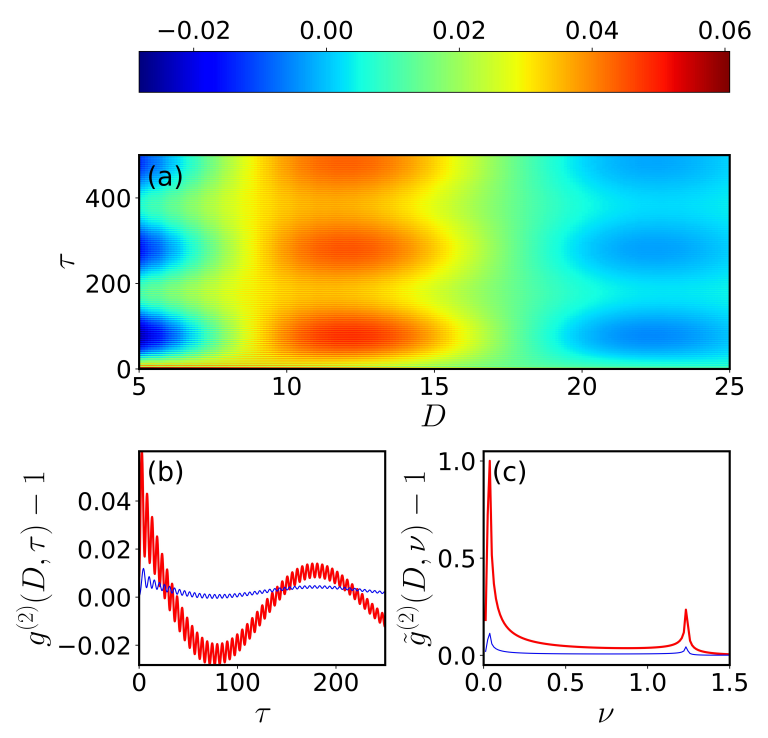

FIG. C1. (color online) Density-density correlation. (a) the density-density correlations as a function of $D$ and $\tau$, when $R=15$ and $\alpha=7.7$. Correlations at $D=5$ (thick red) and 25 (thin blue) are shown in (b). The corresponding Fourier spectrum of the correlation function is shown in (c). In the Fourier spectra, the peaks at lower and higher frequencies are due to the excitation of roton and maxon modes.

the fluctuation when $\tau \rightarrow \infty$,

$$
\frac{\sqrt{N} \Delta n_{\mathrm{c}}^{\infty}}{n} \approx \sqrt{\frac{2 \Gamma \pi^{2}\left[1+\pi^{2} \alpha(6 \sqrt{3}+\pi \alpha C)\right]}{27 R}}
$$

with the constant $C=\left[4 \sqrt{3} \pi-3 \log \left(\frac{27}{16}\right)\right]$. The approximation result shows that fluctuations of the condensate decreases (increases) with increasing $R(\alpha)$. In Fig. B1(b) and (c), numerical and approximate results are both shown. The two calculations agree when $\alpha$ is small or $R$ is large, where the depletion and fluctuation are both small. Though large discrepancy is found when $\alpha$ is large or $R$ is small, the trend found from both numerical and analytical calculations are the same.

\section{Appendix C: Density-Density Correlation}

Lastly we evaluate the density-density correlation function $[10,11]$

$$
g^{(2)}(\mathbf{r}, t)=\sum_{\mathbf{k}, \mathbf{k}^{\prime}, \mathbf{q}} e^{i \mathbf{k} \cdot \mathbf{r}} \frac{1}{\Omega^{2}}\left\langle\hat{a}_{\mathbf{k}+\mathbf{q}}^{\dagger}(t) \hat{a}_{\mathbf{k}}(t) \hat{a}_{\mathbf{k}^{\prime}-\mathbf{q}}^{\dagger}(t) \hat{a}_{\mathbf{k}^{\prime}}(t)\right\rangle
$$

Within the Bogoliubov transformation, this can then be expressed in terms of the condensate density as $\left\langle 1 / \Omega^{2} \sum_{\mathbf{k}, \mathbf{k}^{\prime}} \hat{a}_{\mathbf{k}+\mathbf{q}}^{\dagger}(t) \hat{a}_{\mathbf{k}}(t) \hat{a}_{\mathbf{k}^{\prime}-\mathbf{q}}^{\dagger}(t) \hat{a}_{\mathbf{k}^{\prime}}(t)\right\rangle=n^{2}+$ $n / \Omega \sum_{\mathbf{k}}\left[4\left|v_{\mathbf{k}}\right|^{2}-u_{\mathbf{k}}^{*} v_{\mathbf{k}}-u_{\mathbf{k}} v_{\mathbf{k}}^{*}\right]$. Defining $D=\left|\mathbf{r}-\mathbf{r}^{\prime}\right| / \zeta$ as 


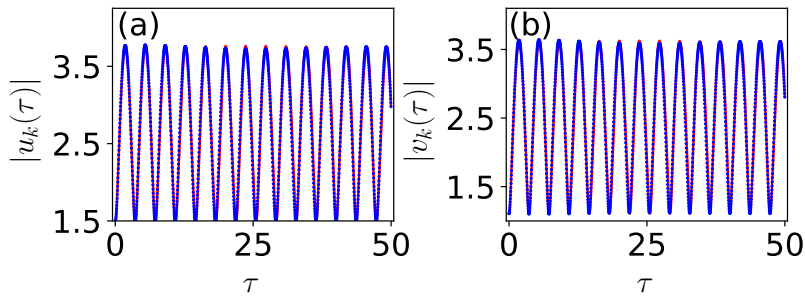

FIG. D1. (color online) Evolution of Bogoliubov amplitudes. Using the same parameters as the thick red curve in Fig. 3(g), the amplitudes $\left|u_{k}(\tau)\right|$ (a) and $\left|v_{k}(\tau)\right|$ (b) are shown for $k=0.3$. The solid red curves corresponds to our self-consistent algorithm while the blue data points are calculated by the rigorous calculation from Ref. [12]

the scaled interatomic distance, the correlation function is given as [11]

$$
\begin{aligned}
g^{(2)}(D, \tau)-1 & =\frac{4 \Gamma}{D} \int_{0}^{\infty} k \mathrm{~d} k \sin (k D) \\
\times & {\left[n_{k}-\operatorname{Re}\left[u_{k}^{*}(\tau) v_{k}(\tau)\right]\right] . }
\end{aligned}
$$

We see from Fig. C1(a) that the correlations immediately develop both slow and fast oscillations. The slow oscillation corresponds to the excitation of roton modes, when $\gamma_{\mathrm{r}}$ is small. The fast oscillations attributed to the maxon occupation are more easily observed when looking at a specific value of $D$ [Fig. C1(b)]. The corresponding Fourier transformation $\tilde{g}^{(2)}(D, \nu)-1$ clearly show the associated frequency peaks. When the distance $D<R$, $g^{(2)}(D, \tau)-1$ oscillates with large amplitudes and can have negative values, i.e. strong repulsive interactions lead to anti-correlations. Around the soft-core radius, the correlations are positive, and reach their maximal values. When $D \gg R$, the correlations tend to zero at large times.

\section{Appendix D: Self-consistent calculation comparison}

The dynamical condensate density is found by calculating the quantum depletion self-consistently. In our calculation we have chosen parameters such that quantum depletion is small in the initial state and also in the dynamical evolution. With this condition at hand, the calculation is carried out by treating $n_{\mathrm{c}}$ as an adiabatically changing parameter. Here a crucial step in the derivation of Eq. (9) of the main text is that we assume $n_{\mathrm{c}}=n$ during the first iteration. With this crude approximation, Eq. (7) can be integrated, leading to Eq. (9). After the first iteration, the value of $n_{\mathrm{c}}$ will be updated and then reinserted into the next iteration in Eq. (9). As the corrected $n_{\mathrm{c}}$ is still very close to $n$, we directly iterate Eq. (9) instead of solving Eq. (7) numerically.

A more rigorous approach was presented by Ref. [12] and should be followed in the event of large quantum depletion. Due to $n_{\mathrm{c}} \approx n$, we can replace the phase term

$$
\phi=\int_{0}^{t} \mathrm{~d} t^{\prime} n_{\mathrm{c}}\left(t^{\prime}\right)
$$

in Ref. [12] with $n_{c} t$ in our calculations. These two calculations appear to agree well when using the parameter regimes considered in the work. Some examples are given in Fig. D1 where we show the dynamical evolution of $u_{k}(t)$ and $v_{k}(t)$ obtained by the two different calculations. The blue curves are obtained from the rigorous approach used in Ref. [12] and red using our calculations.
[1] E. L. Bolda, E. Tiesinga, and P. S. Julienne, Phys. Rev. A 66, 013403 (2002).

[2] D. Jaksch and P. Zoller, Ann. Phys. (N. Y). 315, 52 (2005).

[3] C. J. Pethick and H. Smith, Bose-Einstein Condensation in Dilute Gases (Cambridge University Press, Cambridge, 2008).

[4] L. Pitaevskii and S. Stringari, Bose-Einstein Condensation and Superfluidity (Oxford University Press, 2016).

[5] T. D. Lee, K. Huang, and C. N. Yang, Phys. Rev. 106, 1135 (1957).

[6] R. Lopes, C. Eigen, N. Navon, D. Clément, R. P. Smith, and Z. Hadzibabic, Phys. Rev. Lett. 119, 190404 (2017).

[7] J. Stenger, S. Inouye, M. R. Andrews, H. J. Miesner, D. M. Stamper-Kurn, and W. Ketterle, Phys. Rev. Lett. 82, 2422 (1999).

[8] W. Ketterle, S. Inouye, M. R. Andrews, J. Stenger, H.J. Miesner, and D. M. Stamper-Kurn, Nature 392, 151 (1998).
[9] P. Makotyn, C. E. Klauss, D. L. Goldberger, E. A. Cornell, and D. S. Jin, Nat. Phys. 10, 116 (2014).

[10] G. I. Martone, P. É. Larré, A. Fabbri, and N. Pavloff, Phys. Rev. A 98, 063617 (2018).

[11] S. S. Natu and E. J. Mueller, Phys. Rev. A 87, 053607 (2013).

[12] X. Yin and L. Radzihovsky, Phys. Rev. A 88, 063611 (2013).

[13] A. G. Sykes, J. P. Corson, J. P. D'Incao, A. P. Koller, C. H. Greene, A. M. Rey, K. R. A. Hazzard, and J. L. Bohn, Phys. Rev. A 89, 021601(R) (2014).

[14] B. Kain and H. Y. Ling, Phys. Rev. A 90, 063626 (2014).

[15] C. R. Cabrera, L. Tanzi, J. Sanz, B. Naylor, P. Thomas, P. Cheiney, and L. Tarruell, Science 359, 301 (2018).

[16] L. Santos, G. V. Shlyapnikov, P. Zoller, and M. Lewenstein, Phys. Rev. Lett. 85, 1791 (2000).

[17] U. R. Fischer, Phys. Rev. A 73, 031602(R) (2006).

[18] C. Ticknor, R. M. Wilson, and J. L. Bohn, Phys. Rev. Lett. 106, 065301 (2011). 
[19] T. Lahaye, C. Menotti, L. Santos, M. Lewenstein, and T. Pfau, Rep. Prog. Phys. 72, 126401 (2009).

[20] S. S. Natu, L. Campanello, and S. Das Sarma, Phys. Rev. A 90, 043617 (2014).

[21] R. M. Wilson and S. Natu, Phys. Rev. A 93, 053606 (2016).

[22] Y. Cai, M. Rosenkranz, Z. Lei, and W. Bao, Phys. Rev. A 82, 043623 (2010).

[23] L. Santos, G. V. Shlyapnikov, and M. Lewenstein, Phys. Rev. Lett. 90, 250403 (2003).

[24] L. Chomaz, R. M. Van Bijnen, D. Petter, G. Faraoni, S. Baier, J. H. Becher, M. J. Mark, F. Wächtler, L. Santos, and F. Ferlaino, Nat. Phys. 14, 442 (2018).

[25] I. Lesanovsky, Phys. Rev. Lett. 106, 025301 (2011).

[26] E. Sela, M. Punk, and M. Garst, Phys. Rev. B 84, 085434 (2011).

[27] H. Weimer and H. P. Büchler, Phys. Rev. Lett. 105, 230403 (2010).

[28] W. Li, C. Ates, and I. Lesanovsky, Phys. Rev. Lett. 110, 213005 (2013).

[29] C. Ates, B. Olmos, W. Li, and I. Lesanovsky, Phys. Rev. Lett. 109, 233003 (2012).

[30] M. Saffman, T. G. Walker, and K. Molmer, Rev. Mod. Phys. 82, 2313 (2010).

[31] N. Henkel, F. Cinti, P. Jain, G. Pupillo, and T. Pohl, Phys. Rev. Lett. 108, 265301 (2012).

[32] F. Maucher, N. Henkel, M. Saffman, W. Królikowski, S. Skupin, and T. Pohl, Phys. Rev. Lett. 106, 170401 (2011).

[33] J. Honer, H. Weimer, T. Pfau, and H. P. Büchler, Phys. Rev. Lett. 105, 160404 (2010).

[34] F. Cinti, P. Jain, M. Boninsegni, A. Micheli, P. Zoller, and G. Pupillo, Phys. Rev. Lett. 105, 135301 (2010).

[35] N. Henkel, R. Nath, and T. Pohl, Phys. Rev. Lett. 104, 195302 (2010).

[36] A. Geißler, U. Bissbort, and W. Hofstetter, Phys. Rev. A 98, 063635 (2018).

[37] A. Lauer, D. Muth, and M. Fleischhauer, New J. Phys. 14, 095009 (2012).

[38] Y. Chougale and R. Nath, J. Phys. B. 49, 144005 (2016).

[39] C. Gaul, B. J. DeSalvo, J. A. Aman, F. B. Dunning, T. C. Killian, and T. Pohl, Phys. Rev. Lett. 116, 243001 (2016).

[40] J. B. Balewski, A. T. Krupp, A. Gaj, S. Hofferberth, R. Löw, and T. Pfau, New J. Phys. 16, 063012 (2014).
[41] M. Płodzień, G. Lochead, J. de Hond, N. J. van Druten, and S. Kokkelmans, Phys. Rev. A 95, 043606 (2017).

[42] J. Zeiher, R. Van Bijnen, P. Schauß, S. Hild, J. Y. Choi, T. Pohl, I. Bloch, and C. Gross, Nat. Phys. 12, 1095 (2016).

[43] M. Viteau, M. G. Bason, J. Radogostowicz, N. Malossi, D. Ciampini, O. Morsch, and E. Arimondo, Phys. Rev. Lett. 107, 060402 (2011).

[44] G. Pupillo, A. Micheli, M. Boninsegni, I. Lesanovsky, and P. Zoller, Phys. Rev. Lett. 104, 223002 (2010).

[45] Y. Li, A. Geißler, W. Hofstetter, and W. Li, Phys. Rev. A 97, 023619 (2018).

[46] Y. Y. Jau, A. M. Hankin, T. Keating, I. H. Deutsch, and G. W. Biedermann, Nat. Phys. 12, 71 (2016).

[47] A. L. Gaunt, T. F. Schmidutz, I. Gotlibovych, R. P. Smith, and Z. Hadzibabic, Phys. Rev. Lett. 110, 200406 (2013).

[48] N. Bogoliubov, J. Phys 11, 23 (1947).

[49] Y. Yu, Ann. Phys. 323, 2367 (2008).

[50] G. Natale, R.M.W. van Bijnen, A. Patscheider, D. Petter, M.J. Mark, L. Chomaz, and F. Ferlaino, Phys. Rev. Lett. 123, 050402 (2019).

[51] S. M. Roccuzzo and F. Ancilotto, Phys. Rev. A 99, 041601(R) (2019).

[52] T. Macrì, F. Maucher, F. Cinti, and T. Pohl, Phys. Rev. A 87, 061602(R) (2013).

[53] I. Seydi, S. H. Abedinpour, R. E. Zillich, R. Asgari, and B. Tanatar, Phys. Rev. A 101, 013628 (2020).

[54] Z. Tian, S. Y. Chä, and U. R. Fischer, Phys. Rev. A 97, 063611 (2018).

[55] A. Griesmaier, J. Werner, S. Hensler, J. Stuhler, and T. Pfau, Phys. Rev. Lett. 94, 160401 (2005).

[56] S. S. Natu and S. Das Sarma, Phys. Rev. A 88, 031604(R) (2013).

[57] H. J. Maris, Rev. Mod. Phys. 49, 341 (1977).

[58] C. E. Campbell, E. Krotscheck, and T. Lichtenegger, Phys. Rev. B 91, 184510 (2015).

[59] K. Beauvois, J. Dawidowski, B. Fåk, H. Godfrin, E. Krotscheck, J. Ollivier, and A. Sultan, Phys. Rev. B 97, 184520 (2018).

[60] T. Lahaye, C. Menotti, L. Santos, M. Lewenstein, and T. Pfau, Rep. Prog. Phys. 72, 126401 (2009).

[61] N. Katz, J. Steinhauer, R. Ozeri, and N. Davidson, Phys. Rev. Lett. 89, 220401 (2002). 